\title{
Lightweight Floating-Point Arithmetic: Case Study of Inverse Discrete Cosine Transform
}

\author{
Fang Fang \\ Department of Electrical and Computer Engineering, Carnegie Mellon University, 5000 Forbes Avenue, Pittsburgh, PA 15213, USA
}

Email:ffang@ece.cmu.edu

Tsuhan Chen

Department of Electrical and Computer Engineering, Carnegie Mellon University, 5000 Forbes Avenue, Pittsburgh, PA 15213, USA Email:tsuhan@ece.cmu.edu

\author{
Rob A. Rutenbar \\ Department of Electrical and Computer Engineering, Carnegie Mellon University, 5000 Forbes Avenue, Pittsburgh, PA 15213, USA \\ Email: rutenbar@ece.cmu.edu
}

Received 15 May 2001 and in revised form 9 May 2002

\begin{abstract}
To enable floating-point (FP) signal processing applications in low-power mobile devices, we propose lightweight floating-point arithmetic. It offers a wider range of precision/power/speed/area trade-offs, but is wrapped in forms that hide the complexity of the underlying implementations from both multimedia software designers and hardware designers. Libraries implemented in $\mathrm{C}++$ and Verilog provide flexible and robust floating-point units with variable bit-width formats, multiple rounding modes and other features. This solution bridges the design gap between software and hardware, and accelerates the design cycle from algorithm to chip by avoiding the translation to fixed-point arithmetic. We demonstrate the effectiveness of the proposed scheme using the inverse discrete cosine transform (IDCT), in the context of video coding, as an example. Further, we implement lightweight floating-point IDCT into hardware and demonstrate the power and area reduction.
\end{abstract}

Keywords and phrases: floating-point arithmetic, customizable bit-width, rounding modes, low-power, inverse discrete cosine transform, video coding.

\section{INTRODUCTION}

Multimedia processing has been finding more and more applications in mobile devices. A lot of effort must be spent to manage the complexity, power consumption, and timeto-market of the modern multimedia system-on-chip (SoC) designs. However, multimedia algorithms are computationally intensive, rich in costly FP arithmetic operations rather than simple logic. FP arithmetic hardware offers a wide dynamic range and high computation precision, yet occupies large fractions of total chip area and energy budget. Therefore, its application on mobile computing chip is highly limited. Many embedded microprocessors such as the StrongARM [1] do not include an FP unit due to its unacceptable hardware cost.

So there is an obvious gap in multimedia system development: software designers prototype these algorithms using high-precision FP operations, to understand how the algorithm behaves, while the silicon designers ultimately im- plement these algorithms into integer-like hardware, that is, fixed-point units. This seemingly minor technical choice actually creates severe consequences: the need to use fixedpoint operations often distorts the natural form of the algorithm, forces awkward design trade-offs, and even introduces perceptible artifacts. Error analysis and word length optimization of fixed-point 2D IDCT, inverse discrete cosine transform, algorithm has been studied in [2], and a tool for translating FP algorithms to fixed-point algorithms was presented in [3]. However, such optimization and translation are based on human knowledge of the dynamic range, precision requirements, and the relationship between algorithm's architecture and precision. This time-consuming and errorprone procedure often becomes the bottleneck of the entire system design flow.

In this paper, we propose an effective solution: lightweight FP arithmetic. This is essentially a family of customizable FP data formats that offer a wider range of precision/power/speed/area trade-offs, but wrapped in forms that 
hide the complexity of the underlying implementations from both multimedia algorithm designers and silicon designers. Libraries implemented in $\mathrm{C}++$ and Verilog provide flexible and robust FP units with variable bit-width formats, multiple rounding modes and other features. This solution bridges the design gap between software and hardware and accelerate the design cycle from algorithm to chip. Algorithm designers can translate FP arithmetic computations transparently to lightweight FP arithmetic and adjust the precision easily to what is needed. Silicon designers can use the standard ASIC or FPGA design flow to implement these algorithms using the arithmetic cores we provide which consume less power than standard FP units. Manual translation from FP algorithms to algorithms can be eliminated from the design cycle.

We test the effectiveness of our lightweight arithmetic library using an H.263 video decoder. Typical multimedia applications working with modest-resolution human sensory data such as audio and video do not need the whole dynamic range and precision that IEEE-standard FP offers. By reducing the complexity of FP arithmetic in many dimensions, such as narrowing the bit-width, simplifying the rounding methods and the exception handling, and even increasing the radix, we explore the impact of such lightweight arithmetic on both the algorithm performance and the hardware cost.

Our experiments show that for the H.263 video decoder, FP representation with less than half of the IEEE standard FP bit-width can produce almost the same perceptual video quality. Specifically, only 5 exponent bits and 8 mantissa bits for a radix-2 FP representation, or 3-exponent bits and 11 mantissa bits for a radix-16 FP representation are all we need to maintain the video quality. We also demonstrate that a simple rounding mode is sufficient for video decoding and offers enormous reduction in hardware cost. In addition, we implement a core algorithm in the video codec, IDCT, into hardware using the lightweight arithmetic unit. Compared to a conventional 32-bit FP IDCT, our approach reduces the power consumption by $89.5 \%$.

The paper is organized as follows. Section 2 introduces briefly the relevant background on FP and fixed-point representations. Section 3 describes our $\mathrm{C}++$ and Verilog libraries of lightweight FP arithmetic and the usage of the libraries. Section 4 explores the complexity reduction we can achieve for IDCT built with our customizable library. Based on the results in this section, we present the implementation of lightweight FP arithmetic units and analyze the hardware cost reduction in Section 5. In Section 6, we compare the area/speed/power of a standard FP IDCT, a lightweight FP IDCT, and a fixed-point IDCT. Concluding remarks follow in Section 7.

\section{BACKGROUND}

\subsection{Floating-point representation versus fixed-point representation}

There are two common ways to specify real numbers: FP and fixed-point representations. FP can represent numbers on an

$$
\begin{aligned}
& \begin{array}{|c|c|c|}
\hline 1 & \multicolumn{1}{c}{8} & 23 \\
\hline s & \exp & \text { frac } \\
\hline
\end{array} \\
& \text { FP value: }(1)^{s} 2^{\exp -\text { bias }} \cdot 1 \text {. frac (The leading } 1 \text { is implicit) } \\
& 0 \leq \exp \leq 255 \text {, bias }=127
\end{aligned}
$$

FIGURE 1: FP number representation.

\begin{tabular}{|c|c|}
\hline 16 & 16 \\
\hline int & frac \\
\hline
\end{tabular}

FIGURE 2: Fixed-point number representation.

exponential scale and is reputed for a wide dynamic range. The date format consists of three fields: sign, exponent, and fraction (also called mantissa), as shown in Figure 1.

Dynamic range is determined by the exponent bit-width, and resolution is determined by the fraction bit-width. The widely adopted IEEE single FP standard [4] uses an 8-bit exponent that can reach a dynamic range roughly from $2^{-126}$ to $2^{127}$, and a 23-bit fraction that can provide a resolution of $2^{\exp -127} \cdot 2^{-23}$, where exp stands for value represented by the exponent field.

In contrast, the fixed-point representation is on a uniform scale, that is, essentially the same as the integer representation, except for the fixed radix point. For instance (see Figure 2), a 32-bit fixed-point number with a 16-bit integer part and a 16-bit fraction part can provide a dynamic range of $2^{-16}$ to $2^{16}$ and a resolution of $2^{-16}$.

When prototyping algorithms with $\mathrm{FP}$, programmers do not have to concern about dynamic range and precision, because IEEE standard FP provides more than necessary for most general applications. Hence, float and double are standard parts of programming languages like $\mathrm{C}++$, and are supported by most compilers. However, in terms of hardware, the arithmetic operations of FP need to deal with three parts (sign, exponent, fraction) individually, which adds substantially to the complexity of the hardware, especially in the aspect of power consumption, while fixed-point operations are almost as simple as integer operations. If the system has a stringent power budget, then the application of FP units has to be limited, and on the other hand, a lot of manual work is spent in implementing and optimizing the fixed-point algorithms to provide the necessary dynamic range and precision.

\subsection{IEEE-754 floating-point standard}

IEEE-754 is a standard for binary FP arithmetic [4]. Since our later discussion about the lightweight FP is based on this standard, we give a brief review of its main features in this section.

\section{Data format}

The standard defines two primary formats, single precision (32 bits) and double precision (64 bits). The bit-widths of three fields and the dynamic range of single and doubleprecision FP are listed in Table 1. 
TABLE 1: IEEE FP number format and dynamic range.

\begin{tabular}{lcccccc}
\hline Format & Sign & Exp & Frac & Bias & Max & Min \\
\hline Single & 1 & 8 & 23 & 127 & $3.4 \cdot 10^{38}$ & $1.4 \cdot 10^{-45}$ \\
\hline Double & 1 & 11 & 52 & 1023 & $1.8 \cdot 10^{308}$ & $4.9 \cdot 10^{-324}$
\end{tabular}

\begin{tabular}{|l|c|c|}
\multicolumn{1}{c}{1} & \multicolumn{1}{c}{8} & 23 \\
\hline$s$ & 00000000 & frac $\neq 0$ \\
\hline
\end{tabular}

FIGURE 3: Denormalized number format.

\section{Rounding}

The default rounding mode is round-to-nearest. If there is a tie for the two nearest neighbors, then it is rounded to the one with the least significant bit as zero. Three user selectable rounding modes are: round-toward $+\infty$, round-toward $-\infty$, and round-toward-zero (or called truncation).

\section{Denormalization}

Denormalization is a way to allow gradual underflow. For normalized numbers, because there is an implicit leading 1 , the smallest positive value is $2^{-126} \cdot 1.0$ for single precision (it is not $2^{-127} \cdot 1.0$ because the exponent with all zeros is reserved for denormalized numbers). Values below this can be represented by a so-called denormalized format (Figure 3) that does not have the implicit leading 1 .

The value of the denormalized number is $2^{-126} \cdot 0$. frac and the smallest representable value is hence scaled down to $2^{-149}\left(2^{-126} \cdot 2^{-23}\right)$. Denormalization provides graceful degradation of precision for computations on very small numbers. However, it complicates hardware significantly and slows down the more common normalized cases.

\section{Exception handling}

There are five types of exceptions defined in the standard: invalid operation, division by zero, overflow, underflow, and inexact. As shown in Figure 4, some bit patterns are reserved for these exceptions. When numbers simply cannot be represented, the format returns a pattern called $\mathrm{NaN}$, not-anumber, with information about the problem. NaNs provide an escape mechanism to prevent system crashes in case of invalid operations. In addition to assigning specific $\mathrm{NaN}$ bit patterns, some status flags and trapping signals are used to indicate exceptions.

From the above review, we can see that IEEE standard FP arithmetic has a strong capability to represent real numbers as accurately as possible and is very robust when exceptions occur. However, if the FP arithmetic unit is dedicated to a particular application, the IEEE-mandated 32 or 64-bit long bit-width may provide more precision and dynamic range than needed, and many other features may be unnecessary as well.

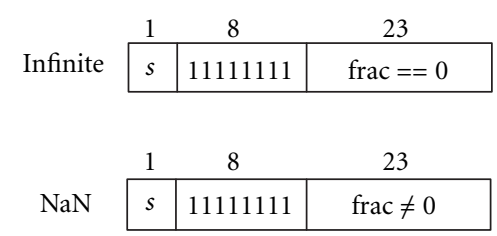

$\mathrm{NaN}$ is assigned when some invalid operations occur, like $(+\infty)+(-\infty), 0 * \infty, 0 / 0, \ldots$ etc.

FIgURE 4: Infinite and NaN representations.

\section{CUSTOMIZABLE LIGHTWEIGHT FLOATING-POINT LIBRARY}

The goal of our customizable lightweight FP library is to provide more flexibility than IEEE standard FP in bit-width, rounding and exception handling. We created matched $\mathrm{C}++$ and Verilog FP arithmetic libraries that can be used during algorithm/circuit simulation and circuit synthesis. With the $\mathrm{C}++$ library, software designers can simulate the algorithms with lightweight arithmetic and decide the minimal bit-width, rounding mode, and so forth, according to the numerical performance. Then with the Verilog library, hardware designers can plug in the parameterized FP arithmetic cores into the system and synthesize it to the gate-level circuit. Our libraries provide a way to move the FP design choices (bit-width, rounding, ...) upwards to the algorithm design stage and better predict the performance during early algorithm simulation.

\subsection{Easy-to-use $\mathrm{C}++$ class Cmufloat for algorithm designers}

Our lightweight FP class is called Cmufloat and implemented by overloading existing $\mathrm{C}++$ arithmetic operators $(+,-, *, /, \ldots)$. It allows direct operations, including assignment between Cmufloat and any $\mathrm{C}++$ data types except char. The bit-width of Cmufloat varies from 1 to 32 including sign, fraction, and exponent bits and is specified during the variable declaration. Three rounding modes are supported: round-to-nearest, Jamming, and truncation, one of which is chosen by defining a symbol in an appropriate configuration file. Explanation of our rounding modes is presented in detail later. In Figure 5, we summarize the operators of Cmufloat and give some examples of using it.

Our implementation of lightweight FP offers two advantages. First, it provides a transparent mechanism to embed Cmufloat numbers in programs. As shown in the example, designers can use Cmufloat as a standard $\mathrm{C}++$ data type. Therefore, the overall structure of the source code can be preserved and a minimal amount of work is spent in translating a standard FP program to a lightweight FP program. Second, the arithmetic operators are implemented by bit-level manipulation, which carefully emulates the hardware implementation. We believe the correspondence between software and hardware is more exact than previous work $[5,6]$. These other approaches appear to have implemented the operators by simply quantizing the result of standard FP operations 


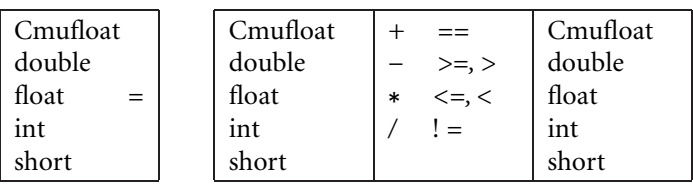

(a) Operators with Cmufloat.

\begin{tabular}{|ll|}
\hline Cmufloat $<14,5>a=0.5 ;$ & // 14-bit fraction and 5-bit exponent \\
Cmufloat $<>b=1.5 ;$ & // Default is IEEE-standard float \\
Cmufloat $<18,6>c[2] ;$ & // Define an array \\
float fa; & \\
& \\
$c[1]=a+b ;$ & \\
$\mathrm{fa}=a * b ;$ & // Assign the result to float \\
$c[2]=\mathrm{fa}+c[1] ;$ & // Operation between float and Cmufloat \\
cout $<<c[2] ;$ & // I/O stream \\
func(a); & // Function call \\
\hline
\end{tabular}

(b) Examples of Cmufloat.

FIGURE 5: Operators and examples of Cmufloat.

into limited bits. This approach actually has more bit-width for the intermediate operations, while our approach guarantees that results of all operations, including the intermediate results, are consistent with the hardware implementation. Hence, the numerical performance of the system during the early algorithm simulation is more trustworthy.

\subsection{Parameterized Verilog library for silicon designers}

We provide a rich set of lightweight FP arithmetic units (adders, multipliers) in the form of parameterized Verilog. First, designers can choose implementations according to the rounding mode and the exception handling. Then they can specify the bit-width for the fraction and exponent by parameters. With this library, silicon designers are able to simulate the circuit at the behavioral level and synthesize it into a gate-level netlist. The availability of such cores makes possible a wider set of design trade-offs (power, speed, area, accuracy) for multimedia tasks.

\section{REDUCING THE COMPLEXITY OF FLOATING-POINT ARITHMETIC FOR A VIDEO CODEC IN MULTIPLE DIMENSIONS}

Most multimedia applications process modest-resolution human sensory data, which allows hardware implementation to use low-precision arithmetic computations. Our work aims to find out how much the precision and the dynamic range can be reduced from the IEEE standard FP without perceptual quality degradation. In addition, the impacts of other features in IEEE standard, such as the rounding mode, denormalization, and the radix choice are also studied. Specifically, we target the IDCT algorithm in an H.263 video codec, since it is the only module that really uses FP computations in the codec, and also is common in many other media applications, such as image processing, audio compression, and so forth.

In Figure 6, we give a simplified diagram of a video codec. In the decoder side, the input compressed video is put into IDCT after inverse quantization. After some FP computations in IDCT, those DCT coefficients are converted to pixel values that are the differences between the previous frame and the current frame. The last step is to get the current frame by adding up the outputs of IDCT and the previous frame after the motion compensation.

Considering the IEEE representation of FP numbers, there are five dimensions that we can explore in order to reduce the hardware complexity (Table 2). Accuracy versus hardware cost trade-off is made in each dimension. In order to measure the accuracy quantitatively, we integrate the Cmufloat IDCT into a complete video codec and measure the PSNR (peak-signal-to-noise-ratio) of the decoded video, which reflects the decoded video quality,

$$
\text { PSNR }=10 \log \frac{255^{2}}{\sum_{i=0}^{N}\left(p_{i}-f_{i}\right) / N},
$$

where $N$ is the total number of pixels, $p_{i}$ stands for the pixel value decoded by the lightweight FP algorithm, and $f_{i}$ stands for the reference pixel value of the original video.

\subsection{Reducing the exponent and fraction bit-width}

\section{Reducing the exponent bit-width}

The exponent bit-width determines the dynamic range. Using a 5-bit exponent as an example, we derive the dynamic range in Table 3. Complying with the IEEE standard, the exponent with all $1 \mathrm{~s}$ is reserved for infinity and $\mathrm{NaN}$. With a bias of 15, the dynamic range for a 5-bit exponent is from $2^{-14}$ or $2^{-15}$ to $2^{16}$, depending on the support for denormalization.

In order to decide the necessary exponent bit-width for our IDCT, we collected the histogram information of exponents for all the variables in the IDCT algorithm during a video sequence decoding (see Figure 7). The range of these exponents lies in $[-22,10]$, which is consistent with the theoretical result in [7]. From the dynamic range analysis above, we know that a 5-bit exponent can almost cover such a range except when numbers are extremely small, while a 6-bit exponent can cover the entire range. However, our experiment shows that 5-bit exponent is able to produce the same PSNR as an 8-bit exponent.

\section{Reducing the fraction bit-width}

Reducing the fraction bit-width is the most practical way to lower the hardware cost because the complexity of an integer multiplier is reduced quadratically with decreasing bit-width [8]. On the other hand, the accuracy is degraded when narrowing the bit-width. The influence of decreasing bit-width on video quality is shown in Figure 8.

As we can see in the curve, PSNR remains almost constant across a rather wide range of fraction bit widths, which means that the fraction width does not affect the decoded 


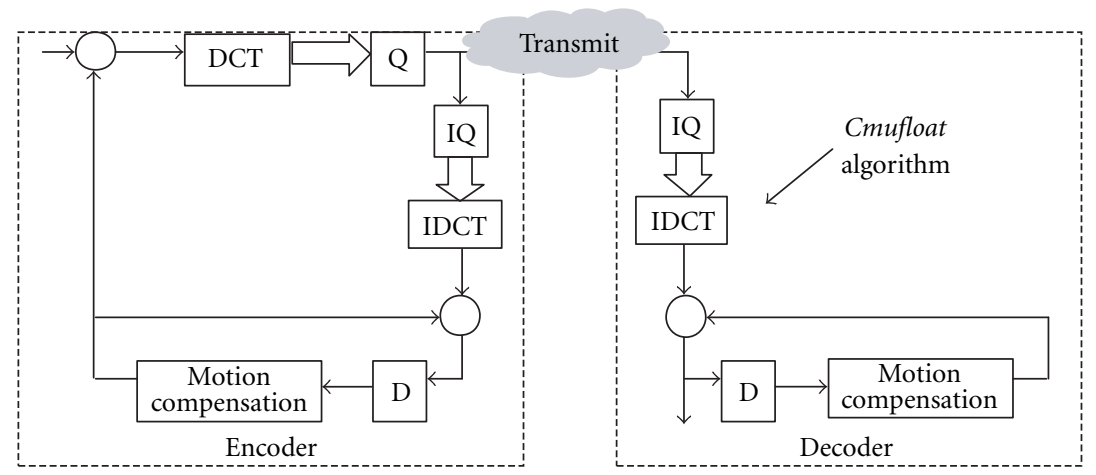

FIGURE 6: Video codec diagram Q: quantizer, IQ: inverse quantizer, D: delay.

TABLE 2: Working dimensions for lightweight FP.

\begin{tabular}{ll}
\hline Dimension & Description \\
\hline Smaller exponent bit-width & $\begin{array}{l}\text { Reduce the number of exponent bits at the expense of dynamic range } \\
\text { Reduce the number of fraction bits at the expense of precision } \\
\text { Simpler rounding mode }\end{array}$ \\
No support for denormalization & $\begin{array}{l}\text { Choose simpler rounding mode at the expense of precision } \\
\text { Do not support denormalization at the expense of precision }\end{array}$ \\
Higher radix & $\begin{array}{l}\text { Increase the implied radix from } 2 \text { to } 16 \text { for the FP exponent (higher radix FP } \\
\text { needs lesponent bits and more fraction bits than radix-2 FP, in order to } \\
\text { achieve the comparable dynamic range and precision) }\end{array}$ \\
\hline
\end{tabular}

TABle 3: Dynamic range of a 5-bit exponent.

\begin{tabular}{lccc}
\hline & Exponent & Value of exp-bias & Dynamic range \\
\hline Biggest exponent & 11110 & 16 & $2^{16}$ \\
\hline $\begin{array}{l}\text { Smallest exponent } \\
\text { (with support for }\end{array}$ & 00001 & -14 & $2^{-14}$ \\
$\begin{array}{l}\text { denormalization) } \\
\text { Smallest exponent } \\
\text { (no support for } \\
\text { denormalization) }\end{array}$ & 00000 & -15 & $2^{-15}$ \\
\hline
\end{tabular}

video quality in this range. The cutoff point where PSNR starts dropping is as small as 8 bits-this is about $1 / 3$ of the fraction-width of an IEEE standard FP. The difference of PSNR between an 8-bit fraction FP and 23-bit fraction is only $0.22 \mathrm{~dB}$, which is almost not perceptible to human eyes. One frame of the video sequence is compared in Figure 9. The top one is decoded by a full precision FP IDCT, and the bottom one is decoded by a 14-bit FP IDCT. From the perceptual quality point of view, it is hard to tell the major difference between these two.

From the above analysis, we can reduce the total bitwidth from 32 bits to 14 bits (1-bit sign +5 -bit exponent + 8-bit fraction), while preserving good perceptual quality. In

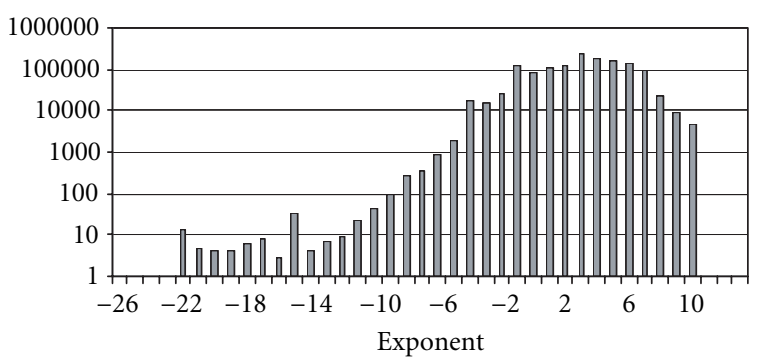

FIGURE 7: Histogram of exponent value.

order to generalize our result, the same experiment is carried on three other video sequences: Akiyo, Stefan, mobile, all of which have around $0.2 \mathrm{~dB}$ degradation in PSNR when 14-bit FP is applied.

\section{The relationship between video compression ratio and the minimal bit-width}

For streaming video, the lower the bit rate, the worse is the video quality. The difference in bit rate is mainly caused by the quantization step size during encoding. A larger step size means coarser quantization and therefore worse video quality. Considering the limited wireless network bandwidth and the low display quality of mobile devices, a relatively low bit rate is preferred to transfer video in this situation. Hence, we 


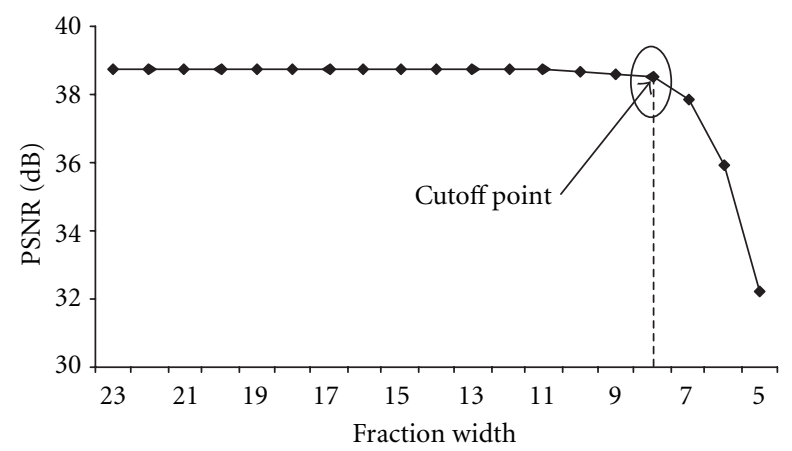

FIGURE 8: PSNR versus fraction width.

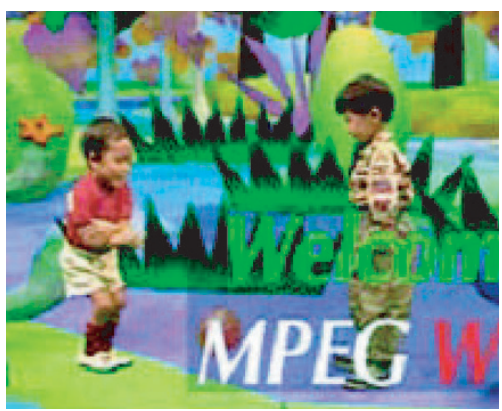

(a) One frame decoded by 32-bit FP 1-bit sign +8 -bit exp +23 -bit fraction.

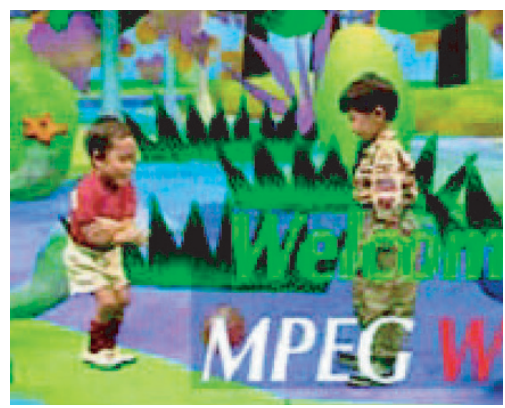

(b) One frame decoded by 14-bit FP 1-bit sign +5 -bit exp +8 -bit fraction.

FIGURE 9: Video quality comparison.

want to study the relationship between compression ratio, or quantization step size and the minimal bit-width.

We compare the PSNR curves obtained by experiments with different quantization step sizes in Figure 10. From the figure, we can see that for a larger quantization step size, the PSNR is lower, but at the same time the curve drops more slowly and the minimal bit-width can be reduced further. This is because coarse quantization can hide more computation error under the quantization noise. Therefore, less computational precision is needed for the video codec using a larger quantization step size.

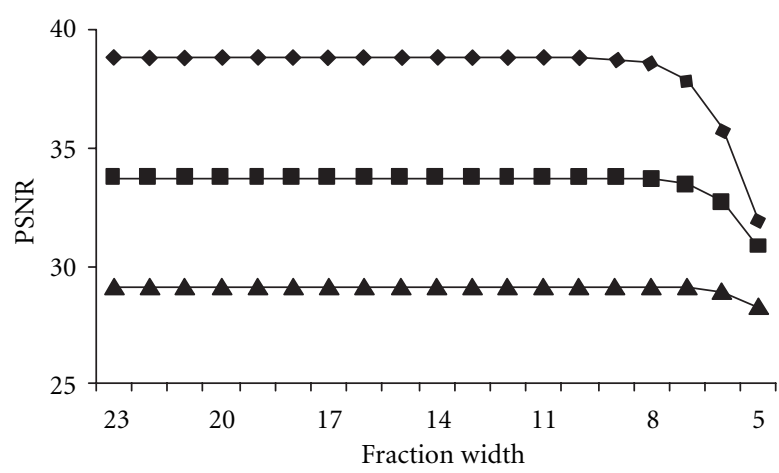

$\longrightarrow$ Quantization step size $=4$
- Quantization step size $=8$
- Quantization step size $=16$

FIgure 10: Comparison of different quantization step.

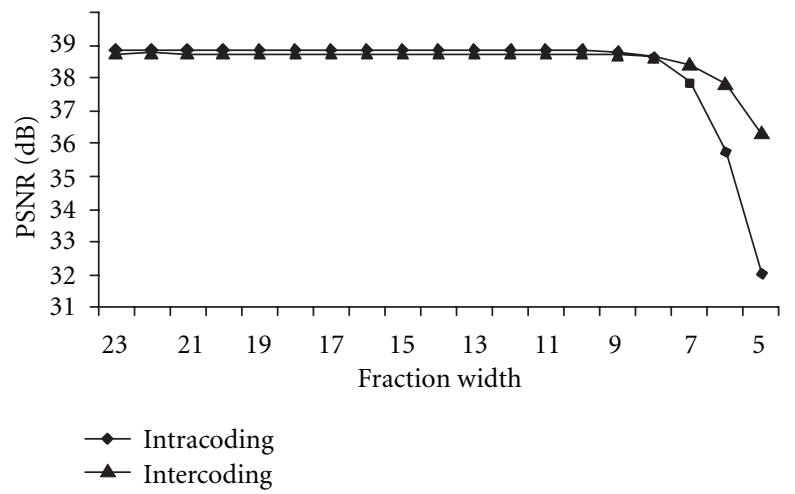

FIGURE 11: Comparison of intracoding and intercoding.

\section{The relationship between inter/intra-coding and the minimal bit-width}

Intercoding refers to the coding of each video frame with reference to the previous video frame. That is, only the difference between the current frame and the previous frame is coded, after motion compensation. Since in most videos, adjacent frames are highly correlated, intercoding provides very high efficiency.

Based on the assumption that video frames have some correlation, for intercoding, the differences coded as the inputs to DCT are typically much smaller than regular pixel values. Accordingly, the DCT coefficients, or inputs to IDCT are also smaller than those in intracoding. One property of FP numbers is that representation error is smaller when the number to be represented is smaller. From Figure 11, the PSNR of intercoding drops more slowly than intracoding, or the minimum bit-width of intercoding can be 1 bit less than intracoding.

However, if the encoder uses the full precision FP IDCT, while the decoder uses the lightweight FP IDCT, then the error propagation effect of intercoding cannot be eliminated. In that case, intercoding does not have the above advantage.

The results in this section demonstrates that some pro- 
grams do not need the extreme precision and dynamic range provided by IEEE standard FP. Applications dealing with the modest-resolution human sensory data can tolerate some computation error in the intermediate or even final results, while giving similar human perceptual results. Some experiments on other applications also agree with this assertion. We also applied Cmufloat to an MP3 decoder. The bit-width can be cut down to 14 bits (1-bit sign + 6-bit exponent + 7-bit fraction) and the noise behind the music is still not perceptible. In the CMU Sphinx application, a speech recognizer, 11-bit FP (1-bit sign + 6-bit exponent + 4-bit fraction) can maintain the same recognition accuracy as 32-bit FP. Such dramatic bit-width reduction offers enormous advantage that may broaden the application of lightweight FP units in mobile devices.

Finally, we note that the numerical analysis and precision optimization in this section can be implemented in a semiautomated way by appropriate compiler support. We can extend an existing $\mathrm{C}++$ compiler to handle lightweight arithmetic operations and assist the process of exploring the precision trade-offs with less programmer intervention. This will unburden designers from translating codes manually into proper limited-precision formats.

\subsection{Rounding modes}

When an FP number cannot be represented exactly, or the intermediate result is beyond the allowed bit-width during computation, then the number is rounded, introducing an error less than the value of the least significant bit. Among the four rounding modes specified by the IEEE FP standard, round-to-nearest, round-to- $(+\infty)$, and round-to- $(-\infty)$ need an extra adder in the critical path, while round-toward-zero is the simplest in hardware, but the least accurate in precision. Since round-to-nearest has the most accuracy, we implement it as our baseline of comparison. There is another classical alternative mode that may have potential in both accuracy and hardware cost: von Neumann [9]. We will discuss these three rounding modes (round-to-nearest, Jamming, round-toward-zero) in detail.

\section{Round-to-nearest}

In the standard FP arithmetic implementation, there are three bits beyond the significant bits that are for intermediate results [10] (see Figure 12). The sticky bit is the logical OR of all bits thereafter.

These three bits participate in rounding in the following way:

$$
\begin{aligned}
& \begin{array}{l}
\begin{array}{l}
b 000 \\
b \cdots \\
b 011
\end{array} \\
\begin{array}{l}
b 100 \\
\begin{array}{l}
101 \\
b \cdots \\
b 111
\end{array}
\end{array}
\end{array} \quad \begin{array}{l}
\text { If } b \text { is } 1 \text {, add } 1 \text { to } b \\
\text { If } b \text { is } 0 \text {, truncate the tail bits }
\end{array} \quad \text { Add } 1 \text { to } b
\end{aligned}
$$

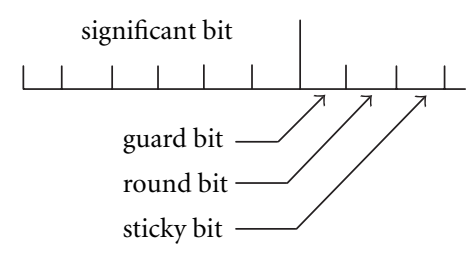

FIGURE 12: Guard/round/sticky bit.

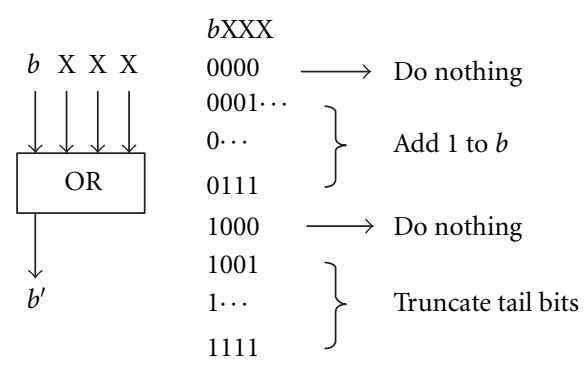

Figure 13: Jamming rounding.

Round-to-nearest is the most accurate rounding mode, but needs some comparison logic and a carry-propagate adder in hardware. Further, since the rounding can actually increase the fraction magnitude, it may require extra normalization steps which cause additional fraction and exponent calculations.

\section{Jamming}

The rule for Jamming rounding is as follows: if $b$ is 1 , then truncate those 3 bits, if $b$ is 0 , and there is a 1 among those 3 bits, then add 1 to $b$, else if $b$ and those 3 bits are all 0 , then truncate those 3 bits. Essentially, it is the function of an OR gate (see Figure 13).

Jamming is extremely simple as hardware, almost as simple as truncation, but numerically more attractive for one subtle but important reason. The rounding created by truncation is biased; the rounded result is always smaller than the correct value. Jamming, by sometimes forcing a 1 into the least significant bit position, is unbiased. The magnitude of Jamming errors is not different from truncation, but the mean of errors is zero. This important distinction was recognized by von Neumann almost 50 years ago [9].

\section{Round-toward-zero}

The operation of round-toward-zero is just truncation. This mode has no overhead in hardware, and it does not have to keep 3 more bits for the intermediate results. So it is much simpler in hardware than the first two modes.

The PNSR curves for the same video sequence obtained using these three rounding modes are shown in Figure 14. Three rounding modes produce almost the same PSNR when the fraction bit-width is more than 8 bits. At the point of 8 bits, the PSNR of truncation is about $0.2 \mathrm{~dB}$ worse than the other two. On the other hand, from the hardware point 


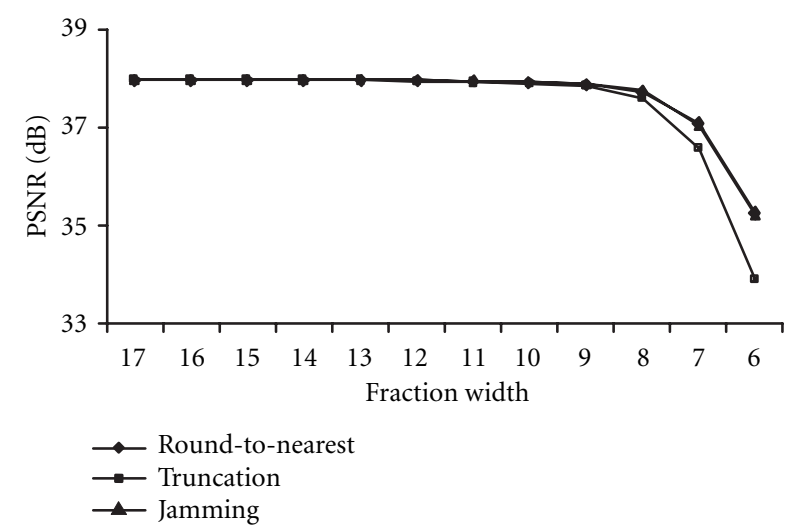

FIGURE 14: Comparison of rounding modes.

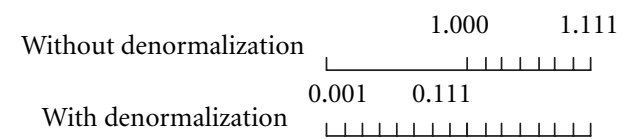

FIGURE 15: Denormalization.

of view, Jamming is much simpler than round-to-nearest and truncation is the simplest among these three modes. So trade-off will be made between quality and complexity among the modes of Jamming and truncation. We will finalize the choice of rounding mode during the hardware implementation section.

\subsection{Denormalization}

The IEEE standard allows for a special set of non-normalized numbers that represent magnitudes very close to zero. We illustrate this in Figure 15 by an example of a 3-bit fraction. Without denormalization, there is an implicit 1 before the fraction, so the actual smallest fraction is 1.000 , while with denormalization, the leading 1 is not enforced so that the smallest fraction is scaled down to 0.001. This mechanism provides more precision for scientific computation with small numbers, but for multimedia applications, especially for video codec, do those small numbers during the computation affect the video quality?

We experimented on the IDCT with a 5-bit exponent Cmufloat representation. 5 bits was chosen to ensure that no overflow would happen during the computation. But from the histogram of Figure 7, there are still some numbers below the threshold of normalized numbers. That means if denormalization is not supported, these numbers will be rounded to zero. However, the experiment shows that the PSNRs with and without denormalization are the same, which means that denormalization does not affect the decoded video quality at all.

\subsection{Higher radix for FP exponent}

The exponent of the IEEE standard FP is based on radix 2 . Historically, there are also systems based on radix 16, for example, the IBM 390 [11]. The advantage of radix 16 lies mainly in fewer types of shifting during prealignment and normalization, which can reduce the shifter complexity in the FP adder and multiplier. We will discuss this issue in Section 5.4 when we discuss hardware implementation in more detail.

The potential advantage of a higher radix such as 16 is that the smaller exponent bit-width is needed for the same dynamic range as the radix-2 $\mathrm{FP}$, while the disadvantage is that the larger fraction bit-width has to be chosen to maintain the comparable precision. We analyze such features in the following.

\section{Exponent bit-width}

The dynamic range represented by $i$-bit exponent is approximately from $\beta^{2^{-(i-1)}}$ to $\beta^{2^{(i-1)}}$ ( $\beta$ is the radix). Assume we use $i$-bit exponent for radix-2 FP and $j$-bit exponent for radix16 FP. If they have the same dynamic range, then $2^{2^{-(i-1)}}=$ $16^{2^{(j-1)}}$, or $j=i-2$. Specifically, if the exponent bit-width for radix-2 is 5 , then only 3 bits are needed for the radix-16 FP to reach approximately the same range.

\section{Fraction bit-width}

The precision of an FP number is mainly determined by the fraction bit-width. But the radix for the exponent also plays a role, due to the way that normalization works. Normalization ensures that no number can be represented with two or more bit patterns in the FP format, thus maximizing the use of the finite number of bit patterns. Radix-2 numbers are normalized by shifting to ensure a leading bit 1 in the most significant fraction bit. IEEE format actually makes this implicit, that is, it is not physically stored in the number.

For radix 16, however, normalization means that the first digit of the fraction, that is, the most significant 4 bits after the radix point, is never 0000 . Hence there are four bit patterns that can appear in the radix-16 fraction (see Table 4). In other words, the radix-16 fraction uses its available bits in a less efficient way, because the leading zeros reduce the number of significant bits of precision. We analyze the loss of significant precisions bits in Table 4 .

The significant bit-width of a radix- $2 i$-bit fraction is $i+1$, while for a radix-16 $j$-bit fraction, the significant bit-width is $j, j-1, j-2$, or $j-3$, with possibility of $1 / 4$, respectively. The minimum fraction bit-width of a radix-16 FP that can guarantee the precision not less than radix-2 must satisfy the following inequality:

$$
\min \{j, j-1, j-2, j-3\} \geq i+1,
$$

so the minimum fraction bit-width is $i+4$. Actually, it can provide more precision than radix-2 FP since $j, j-1, j-2$ are larger than $i+1$.

From previous discussions, we know that 14-bit radix-2 FP (1-bit sign + 5-bit exponent +8 -bit fraction) can produce good video quality. Moving to radix-16, two less exponent bits ( 3 bits) and four more fraction bits ( 12 bits), or 16 bits for total FP can guarantee the comparable video quality. 
TABle 4: Comparison of radix-2 to radix-16.

\begin{tabular}{lccc}
\hline PP format & Normal form of fraction & Range of fraction & Significant bits \\
Radix-2 (i-bit fraction) & $1 . \mathrm{xx} \cdots \mathrm{xx}$ & $1 \leq f<2$ & $i+1$ \\
\hline & $.1 \mathrm{xx} \cdots \mathrm{x}$ & $1 / 2 \leq f<1$ & $j$ \\
Radix-16 ( $j$-bit fraction) & $.01 \mathrm{xx} \cdot \mathrm{x}$ & $1 / 4 \leq f<1 / 2$ & $j-1$ \\
& $.001 \mathrm{x} \cdot \cdots \mathrm{x}$ & $1 / 8 \leq f<1 / 4$ & $j-2$ \\
& $.0001 \mathrm{x} \cdot \mathrm{x}$ & $1 / 16 \leq f<1 / 8$ & $j-3$ \\
\hline
\end{tabular}

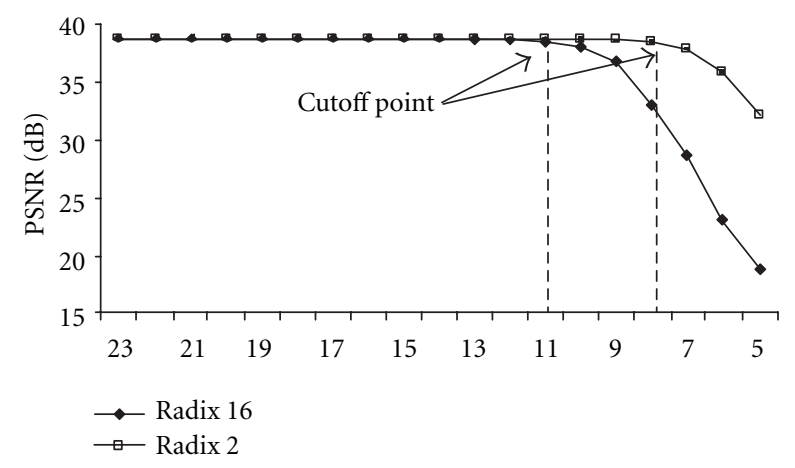

FIgURE 16: Comparison of radix-2 and radix-16.

TABLE 5: 11-bit fraction for radix-16 is sufficient.

\begin{tabular}{lc}
\hline FP format & PSNR \\
\hline Radix-2 (8-bit fraction) & 38.529 \\
\hline Radix-16 (12-bit fraction) & 38.667 \\
\hline Radix-16 (11-bit fraction) & 38.536 \\
\hline Radix-16 (10-bit fraction) & 38.007 \\
\hline
\end{tabular}

Since the minimum fraction bit-width is derived from the worst case analysis, it could be reduced further from the perspective of average precision. Applying radix-16 Cmufloat to the video decoder, we can see that the cutoff point actually is 11 bits, not 12 bits for fraction width (Figure 16 and Table 5).

After discussion in each working dimension, we summarize the lightweight FP design choices for the H.263 video codec in the following:

Data format: 14-bit radix-2 FP (5-bit exponent + 8-bit fraction) or 15-bit radix-16 FP (3-bit exponent + 11-bit fraction).

Rounding: Jamming or truncation.

Denormalization: not supported.

The final choice of data format and rounding mode are made in Section 5 according to the hardware cost.

In all discussions in this section, we use PSNR as a measurement of the algorithm. However, we need to mention that there is an IEEE standard specifying the precision requirement for $8 \times 8$ DCT implementation [12]. In the stan- dard, it has the following specification:

$$
\text { omse }=\frac{\sum_{i=0}^{7} \sum_{j=0}^{7} \sum_{k=0}^{10000} e_{k}^{2}(i, j)}{64 \times 10000} \leq 0.02
$$

(where omse is the overall-mean-square-error, $e_{k}$ is the pixel difference between reference and proposed IDCT, and $i, j$ are the position of the pixel in the $8 \times 8$ block).

PSNR specification can be derived from omse: PSNR $=10 \log _{10}\left(255^{2} /\right.$ omse $) \geq 65.1 \mathrm{~dB}$, which is too tight for videos/images displayed by mobile devices. The other reason we did not choose this standard is that it uses the uniform distributed random numbers as input pixels that eliminate the correlation between pixels and enlarge the FP computation error. We also did experiments based on the IEEE standard. It turns out that around 17-bit fraction is required to meet all the constraints. From PSNR curves in this section, we know that PSNR almost keeps constant for fraction width from 17 bits down to 9 bits. The experimental results support our claim very well that IEEE standard specifications for IDCT is too strict for encoding/decoding real video sequences.

\section{HARDWARE IMPLEMENTATION OF LIGHTWEIGHT FP ARITHMETIC UNITS}

FP addition and multiplication are the most frequent FP operations. A lot of work has been published about IEEE compliant FP adders and multipliers, focusing on reducing the latency of the computation. In IBM RISC System/6000, leading zero anticipator is introduced in the FP adder [13]. The SNAP project proposed a two-path approach in the $\mathrm{FP}$ adder [14]. However, the benefit of these algorithms is not significant and the penalty in area is not ignorable when the bitwidth is very small. In this section, we present the structure of the FP adder and multiplier appropriate for narrow bitwidth and study the impact of different rounding/exception handling/radix schemes.

Our design is based on Synopsys Designware library and STMicroelectronics $0.18 \mu \mathrm{m}$ technology library. The area and latency are measured on gate-level circuit by Synopsys DesignCompiler, and the power consumption is measured by Cadence VerilogXL simulator and Synopsys DesignPower. 


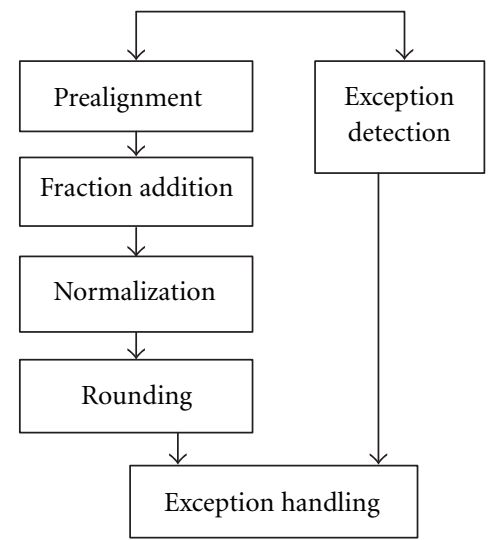

(a) Adder.

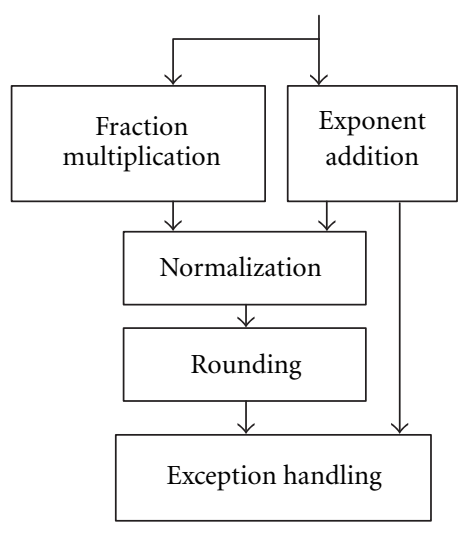

(b) Multiplier.

FIGURE 17: Diagram of FP, the adder and multiplier.

\subsection{Structure}

As shown in Figure 17, we take the most straightforward toplevel structure for the FP adder and multiplier. The tricks reducing the latency are not adopted because firstly the adder and multiplier can be accelerated easily by pipelining, and secondly those tricks increase the area by a large percentage in the case of narrow bit width.

\section{Shifter}

The core component in the prealignment and normalization is a shifter. There are three common architectures for shifter:

$N$-to-1 Mux shifter is appropriate when $N$ is small.

Logarithmic shifter [15] uses $\log (N)$ stages and each stage handles a single, power-of-2 shifts. This architecture has compact area, but the timing path is long when $N$ is big.

Fast two-stage shifter is used in IBM RISC System/6000 [16]. The first stage shifts $(0,4,8,12, \ldots)$ bit positions, and the second stage shifts $(0,1,2,3)$ bit position.

The comparison of these three architectures over different bit-widths is shown in Figure 18. It indicates that for narrow bit-width $(8 \sim 16)$, logarithmic shifter is the best choice considering both of area and delay, but when the
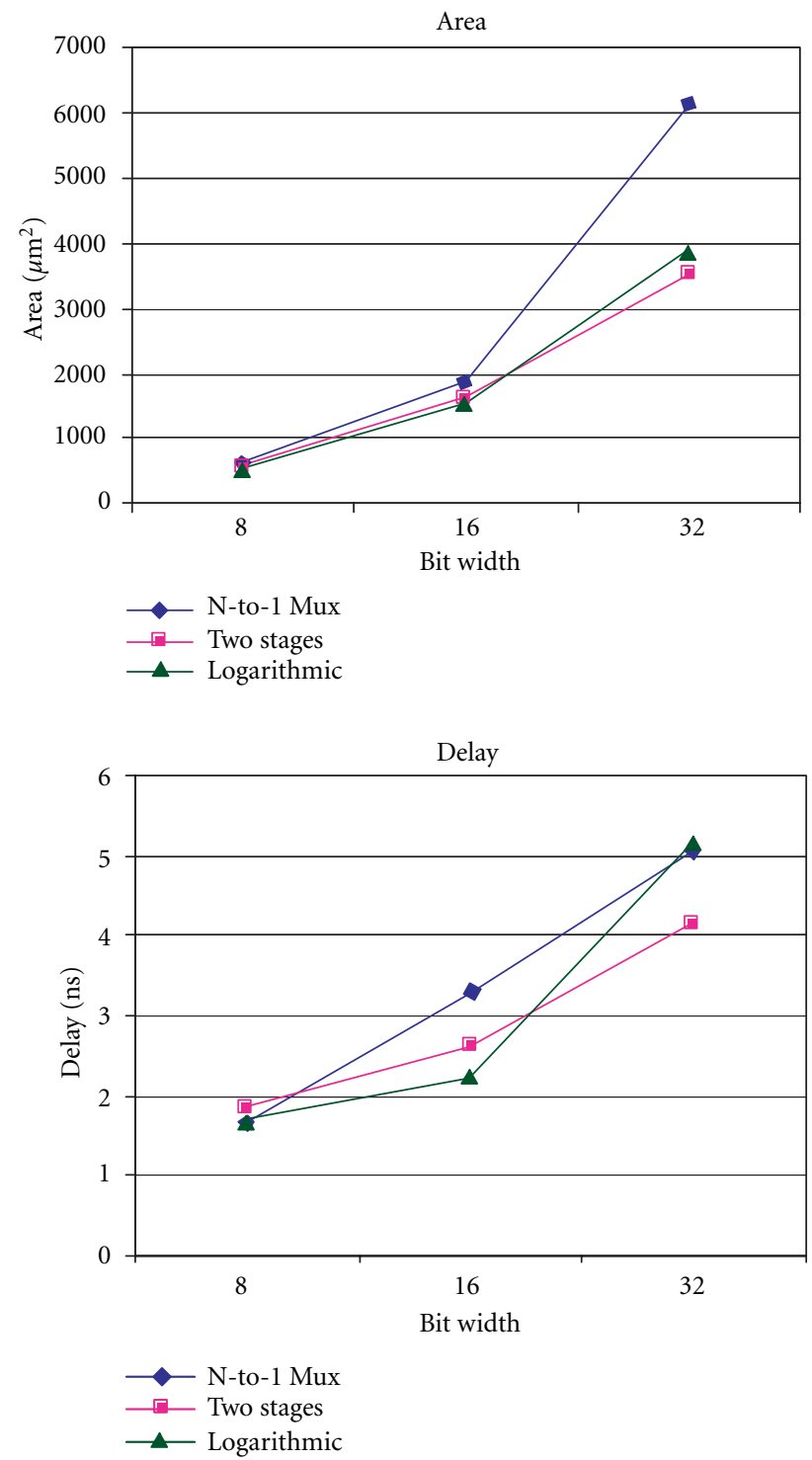

FIGURE 18: Area and delay comparisons of three shifter structures.

bit-width is increased to a certain level, two-stage shifter become best.

\subsection{Rounding}

In our lightweight FP arithmetic operations, round-tonearest is not considered because of the heavy hardware overhead. Jamming rounding demonstrates similar performance as round-to-nearest in the example of video codec. But it still has to keep three more bits in each stage in the FP adder, which becomes significant, especially for narrow bitwidth cases. The other candidate is round-toward-zero because its performance is close to Jamming rounding at the cutoff point. Table 6 shows the reduction in both of the area and delay when changing rounding mode from Jamming to round-toward-zero. Since $15 \%$ reduction in the area of FP adder can be obtained, we finally choose truncation as the rounding mode. 
TABLE 6: Comparison of rounding modes.

\begin{tabular}{lcc}
\hline Rounding mode & Area $\left(\mathrm{um}^{2}\right)$ & Delay $(\mathrm{ns})$ \\
\hline Jamming & 7893 & 5.8 \\
Truncation & $7741(-1.9 \%)$ & $5.71(-1.6 \%)$ \\
\hline
\end{tabular}

(a) 14-bit FP adder.

\begin{tabular}{lcc}
\hline Rounding mode & Area $\left(\mathrm{um}^{2}\right)$ & Delay (ns) \\
\hline Jamming & 8401 & 10.21 \\
Truncation & $7123(-15 \%)$ & $9.43(-7.6 \%)$ \\
\hline
\end{tabular}

(b) 14-bit FP multiplier.

TABLE 7: Comparison of exception handling.

\begin{tabular}{lcc}
\hline Exception handling & Area $\left(\mathrm{um}^{2}\right)$ & Delay $(\mathrm{ns})$ \\
\hline Full & 8401 & 10.21 \\
Partial & $7545(-10 \%)$ & $9.26(-9.3 \%)$ \\
\hline
\end{tabular}

(a) 14-bit FP adder.

\begin{tabular}{lcc}
\hline Exception handling & Area $\left(\mathrm{um}^{2}\right)$ & Delay $(\mathrm{ns})$ \\
\hline Full & 7893 & 5.8 \\
Partial & $7508(-4.9 \%)$ & $4.62(-20 \%)$ \\
\hline
\end{tabular}

(b) 14-bit multiplier.

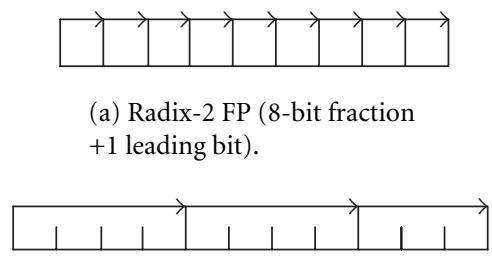

(b) Radix-16 FP (11-bit fraction).

FIGURE 19: Shifting positions for radix-2 and radix-16 FP.

\subsection{Exception handling}

For an IEEE compliant FP arithmetic unit, a large portion of hardware in critical timing path is dedicated for rare exceptional cases, for example, overflow, underflow, infinite, $\mathrm{NaN}$ and so forth. If the exponent bit-width is enough to avoid overflow, then infinite and $\mathrm{NaN}$ will not occur during computation. Then in the FP adder and multiplier diagram (Figure 17), exception detection is not needed and only underflow is detected in exception handling. As a result, the delay of the FP adder and multiplier is reduced by $9.3 \%$ and $20 \%$, respectively, using partial exception handling.

\subsection{Radix}

The advantage of higher radix FP is less complex in the shifter. From Section 4, we know that in video codec, the precision of radix-16 FP with 11-bit fraction is close to radix-2
TABLE 8: Comparison of radix 2 and radix 16.

\begin{tabular}{ccc}
\hline Radix & Area $\left(\mathrm{um}^{2}\right)$ & Delay (ns) \\
\hline 2 & 8401 & 10.21 \\
16 & $7389(-12 \%)$ & $8.48(-17 \%)$ \\
\hline
\end{tabular}

(a) FP adder.

\begin{tabular}{ccc}
\hline Radix & Area $\left(\mathrm{um}^{2}\right)$ & Delay (ns) \\
\hline 2 & 7893 & 5.8 \\
16 & $11284(+43 \%)$ & $6.62(+14 \%)$ \\
\hline
\end{tabular}

(b) FP multiplier.

TABLE 9

\begin{tabular}{lccc}
\hline Data format & Area $\left(\mathrm{um}^{2}\right)$ & Delay $(\mathrm{ns})$ & Power $(\mathrm{mW})$ \\
\hline Lightweight & 10666 & 5.67 & 16.5 \\
IEEE standard & 51830 & 14.5 & 100.1
\end{tabular}

(a) Comparison of lightweight FP and IEEE FP-adder.

\begin{tabular}{lccc}
\hline Data format & Area $\left(\mathrm{um}^{2}\right)$ & Delay $(\mathrm{ns})$ & Power $(\mathrm{mW})$ \\
\hline Lightweight & 5206 & 7.49 & 6.9 \\
IEEE standard & 19943 & 21.7 & 29.3 \\
\hline
\end{tabular}

(b) Comparison of lightweight FP and IEEE FP-multiplier.

FP with 8-bit fraction. We illustrate the difference in shifter in (Figure 19). The step size of shifting for radix-16 is four and only three shifting positions are needed. Such a simple shifter can be implemented by the structure of 3-to-1 Mux. Although there are 3 more bits in the fraction, FP adder still benefited from higher radix in both area and delay (Table 8).

On the other hand, more fraction width increases the complexity of the FP multiplier. The size of multiplier is increased about quadratically with the bit-width, so only 3 more bits can increase the multiplier's area by $43 \%$ (Table 8 ).

From the table, it is clear that radix-16 is not always better than radix-2. In a certain application, if there are more adders than multipliers, then radix- 16 is a better choice than radix-2. In our IDCT structure, there are 29 adders and 11 multipliers. Therefore, radix-16 is chosen for the implementation of IDCT.

Combining all the optimization strategies in this section, the final 15-bit radix-16 FP adder and multiplier are compared with the IEEE standard compliant single-precision FP adder and multiplier in Table 9. Reducing the bit-width and simplifying the rounding/shifting/exception handling, the power consumption of FP arithmetic unit is cut down to around $1 / 5$ of the IEEE standard FP unit.

Further optimization can be conducted in two directions. One is low-power design approach. As proposed in [17], triple data path in FP adder structure can reduce the power delay product by 16X. The other is transistor level optimization. Shifter and multiplexor designed in transistors 


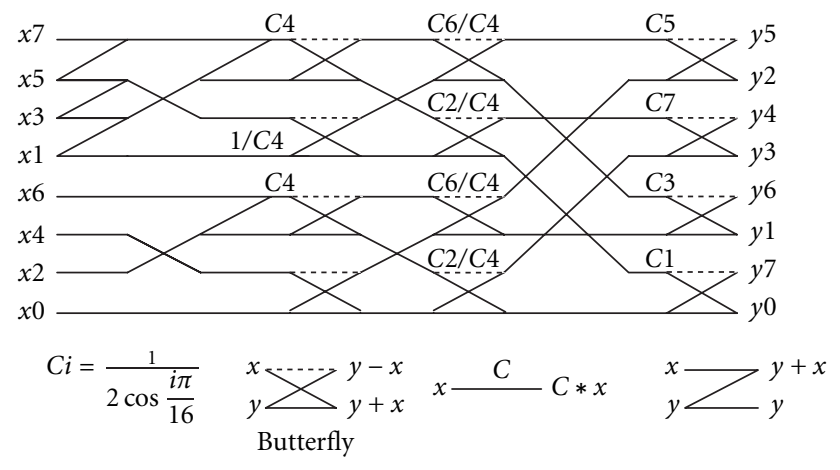

FIgURE 20: Structure of IDCT.

are much smaller and faster than those implemented as logic gates $[15,18]$.

\section{IMPLEMENTATION OF IDCT}

IDCT performs linear transform on an 8-input data set. The algorithm developed in [19] uses minimum resources, 11 multiplications and 29 additions, to compute a onedimensional IDCT (Figure 20).

\subsection{Optimization in butterfly}

In the architecture of IDCT, there are 12 butterflies and each one is composed of two adders. Because these two adders have the same inputs, some operations such as prealignment and negation can be shared. Further, in the butterfly structure, normalization and leading-one detection can be separated into two paths and executed in parallel, which reduce the timing critical path.

Figure 21a is a diagram of the FP adder, and Figure 21b is a diagram of the butterfly with the function of two FP adders. From the figure, we can see that a butterfly is similar to one FP adder in structure except one extra integer adder and some selection logic. Table 10 shows that such butterfly structure saves $37 \%$ area compared with two simple adders.

This reduction is due to the property of FP addition. For a fixed-point butterfly, no operations can be shared.

\subsection{Results comparison}

We implement the IDCT in 32-bit IEEE FP, 15-bit radix-16 lightweight FP, and fixed-point algorithms (see the comparison in Table 11). In the fixed-point implementation, we preserve 12-bit accuracy for constants, and the widest bit-width is 24 in the whole algorithm (not fine tuned). From the perspective of power, the lightweight FP IDCT consumes only around $1 / 10$ of the power compared to the IEEE FP IDCT, and is comparable with the fixed-point implementation.

\section{CONCLUSION}

In this paper, we introduce $\mathrm{C}++$ and Verilog libraries of lightweight FP arithmetic, focusing on the most critical arithmetic operators (addition, multiplication), and the

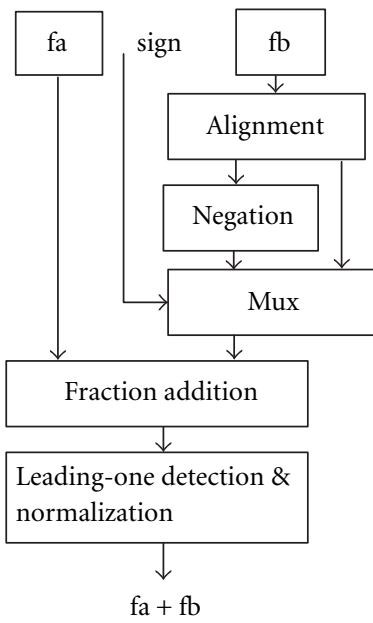

(a) FP adder.

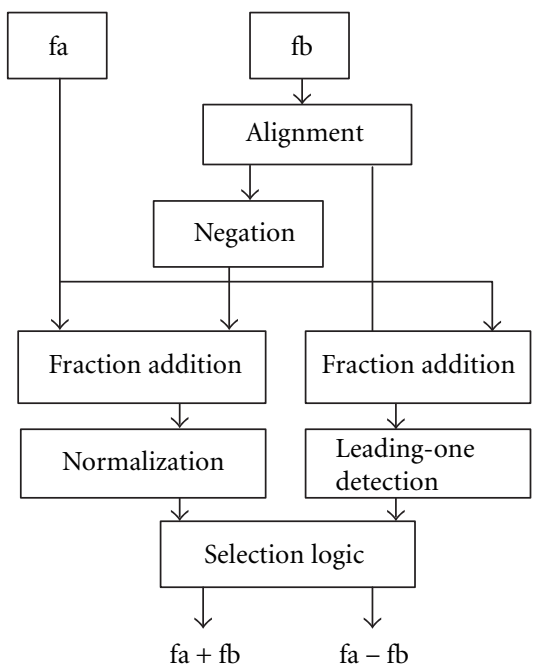

(b) FP butterfly.

Figure 21: Diagrams of a FP adder and a FP butterfly.

most common parameterizations useful for multimedia tasks (bit-width, rounding modes, exception handling, radix). With these libraries, we can easily translate a standard FP program to a lightweight FP program, and explore the system numerical performance versus hardware complexity tradeoff.

An H.263 video codec is chosen to be our benchmark. Such media applications do not need a wide dynamic range and high precision in computations, so the lightweight FP can be applied efficiently. By examining the histogram information of FP numbers and relationship between PSNR and bit-width, we demonstrate that our video codec have almost no quality degradation when more than half of the bit-width in standard FP is reduced. Other features specified in the standard FP, such as rounding modes, exception handling and the radix choice, are also discussed for this partic- 
TABle 10: Comparison of two FP adders and a "butterfly."

\begin{tabular}{lcc}
\hline Structure & Area $\left(\mathrm{um}^{2}\right)$ & Delay $(\mathrm{ns})$ \\
\hline Two FP adders & 10412 & 7.49 \\
Butterfly & 6545 & 8.14 \\
\hline
\end{tabular}

TABLE 11: Comparison of three implementations of IDCT.

\begin{tabular}{lccc}
\hline Implementation & Area $\left(\mathrm{um}^{2}\right)$ & Delay $(\mathrm{ns})$ & Power $(\mathrm{mW})$ \\
\hline IEEE FP & 926810 & 111 & 1360 \\
Lightweight FP & 216236 & 46.75 & 143 \\
Fixed-point & 106598 & 36.11 & 110 \\
\hline
\end{tabular}

ular application. Such optimization offers huge reduction in hardware cost. In the hardware implementation of IDCT, we combined two FP adders in a butterfly (basic component of IDCT), which further reduced the hardware cost. At last, we show that power consumption of the lightweight FP IDCT is only $10.5 \%$ of the standard FP IDCT, and comparable to the fixed-point IDCT.

\section{ACKNOWLEDGMENT}

This work was funded in part by the Pittsburgh Digital Green house, and the Semiconductor Research Corporation.

\section{REFERENCES}

[1] D. Dobberpuhl, "The design of a high performance low power microprocessor," in International Symposium on Low Power Electronics and Design, pp. 11-16, Montery, Calif, USA, August 1996.

[2] S. Kim and W. Sung, "Fixed-point error analysis and word length optimization of $8 \times 8$ IDCT architectures," IEEE Trans. Circuits and Systems for Video Technology, vol. 8, no. 8, pp. 935-940, 1998.

[3] K. Kum, J. Kang, and W. Sung, “AUTOSCALER for C: An optimizing floating-point to integer $\mathrm{C}$ program converter for fixed-point digital signal processors," IEEE Trans. on Circuits and Systems II: Analog and Digital Signal Processing, vol. 47, no. 9, pp. 840-848, 2000.

[4] The Institute of Electrical and Electronics Engineers, Inc., "IEEE-standard for Binary Floating-Point Arithmetic," ANSI/IEEE Std 754-1985, 1985.

[5] D. M. Samanj, J. Ellinger, E. J. Powers, and E. E. Swartzlander, "Simulation of variable precision IEEE floating point using $\mathrm{C}++$ and its application in digital signal processor design," in Circuits and Systems, Proceedings of the 36th Midwest Symposium on, pp. 1509-1514, 1993.

[6] R. Ignatowski and E. E. Swartzlander, "Creating new algorithm and modifying old algorithms to use the variable precision floating point simulator," in Signals, Systems and Computers, 1994 Conference Record of the 28th Asilomar Conference on, vol. 1, pp. 152-156, 1994.

[7] X. Wan, Y. Wang, and W. H. Chen, "Dynamic range analysis for the implementation of fast transform," IEEE Trans. Cir- cuits and Systems for Video Technology, vol. 5, no. 2, pp. 178180, 1995.

[8] P. C. H. Meier, R. A. Rutenbar, and L. R. Carley, "Exploring multiplier architecture and layout for low power," in Proc. IEEE 1996 Custom Integrated Circuits Conference, pp. 513-516, San Diego, Calif, USA, May 1996.

[9] A. W. Burks, H. H. Goldstine, and J. von Neumann, Preliminary Discussion of the Logical Design of an Electronics Computing Instrument, Computer Structures: Reading and Examples. McGraw-Hill, 1971.

[10] The Institute of Electrical and Electronics Engineers, Inc., "A Proposed Standard for Binary Floating-Point Arithmetic," Draft 8.0 of IEEE Task P754, 1981.

[11] S. F. Anderson, J. G. Earle, R. E. Goldschmidt, and D. M. Powers, "The IBM system 390 model 91: floating point execution unit," IBM Journal of Research and Development, vol. 11, no. 1, pp. 34-53, 1967.

[12] Institute of Electrical and Electronics Engineers, Inc., "IEEEstandard specifications for the implementations of $8 \times 8$ inverse discrete cosine transform," IEEE Std 1180-1990, 1990.

[13] E. Hokenek and R. K. Montoye, "Leading-zero anticipator (LZA) in the IBM RISC system/6000 floating-point execution unit," IBM Journal of Research and Development, vol. 34, no. 1, pp. 71-77, 1990.

[14] S. F. Oberman, H. AL-Twaijry, and M. J. Flynn, "The SNAP project: design of floating point arithmetic units," in Proc. 13th Symposium on Computer Arithmetic, pp. 156-165, Asilomar, Calif, USA, July 1997.

[15] K. P. Acken, M. J. Irwin, and R. M. Owens, "Power comparisons for barrel shifters," in International Symposium on Low Power Electronics and Design, pp. 209-212, Monterey, Calif, USA, August 1996.

[16] R. K. Montoye, E. Hokenek, and S. L. Runyon, "Design of the IBM RISC system/6000 floating-point execution unit," IBM Journal of Research and Development, vol. 34, no. 1, pp. 59-70, 1990.

[17] R. V. K. Pillai, D. Al-Khalili, and A. J. Al-Khalili, "A low power approach to floating point adder design," in Proc. IEEE International Conference on Computer Design: VLSI in Computers and Processors, pp. 178-185, Austin, Tex, USA, October 1997.

[18] J. M. Rabaey, Digital Integrated Circuits: A Design Perspective, Prentice-Hall, New Jersey, USA, 1996.

[19] A. Artieri and O. Colavin, "A chip set core for image compression," IEEE Trans. on Consumer Electronics, vol. 36, pp. 395402, August 1990.

Fang Fang received the B.S. degree in electrical engineering from Southeast University, China in 1999 and the M.S. degree from Carnegie Mellon University, Pittsburgh, Pa in 2001. Currently, she is a Ph.D. candidate in the Center for Silicon System Implementation and the Advanced Multimedia Processing Lab in Electrical and Computer Engineering Department at Carnegie Mellon Univesity. Her research interest includes numerical performance of signal processing algorithms and high level hardware synthesis of DSP algorithms. Her current work focus on automatic design flow of the lightweight floating-point system, and hardware synthesis of WHT and FFT transforms. 
Tsuhan Chen received the B.S. degree in electrical engineering from the National Taiwan University in 1987, and the M.S. and Ph.D. degrees in electrical engineering from the California Institute of Technology, Pasadena, California, in 1990 and 1993, respectively. Since October 1997, Tsuhan Chen has been with the Department of Electrical and Computer Engineering, Carnegie Mellon University, Pitts-

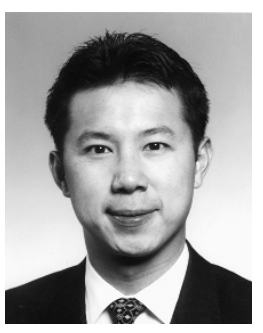
burgh, Pennsylvania, where he is now a Professor. He directs the Advanced Multimedia Processing Laboratory. His research interests include multimedia signal processing and communication, audiovisual interaction, biometrics, processing of $2 \mathrm{D} / 3 \mathrm{D}$ graphics, bioinformatics, and building collaborative virtual environments. From August 1993 to October 1997, he worked in the Visual Communications Research Department, AT\&T Bell Laboratories, Holmdel, New Jersey, and later at AT\&T Labs-Research, Red Bank, New Jersey. Tsuhan helped create the Technical Committee on Multimedia Signal Processing, as the founding chair, and the Multimedia Signal Processing Workshop, both in the IEEE Signal Processing Society. He has recently been appointed as the Editor-in-Chief for IEEE Transactions on Multimedia for the period 2002-2004. He has coedited a book titled "Advances in Multimedia: Systems, Standards, and Networks." He is a recipient of the National Science Foundation Career Award.

Rob A. Rutenbar received the Ph.D. degree from the University of Michigan in 1984, and subsequently joined the faculty of Carnegie Mellon University. He is currently the Stephen J. Jatras Professor of Electrical and Computer Engineering, and (by courtesy) of Computer Science. He is the founding Director of the MARCO/DARPA Center for Circuits, Systems, Software (C2S2),

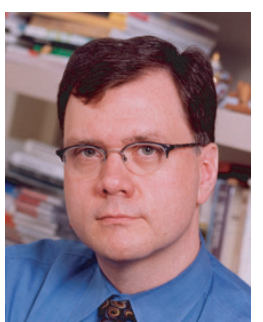
a consortium of U.S. Universities chartered in 2001 to explore long-term solutions for next-generation circuit challenges. His research interests focus on circuit and layout synthesis algorithms for mixed-signal ASICs, and for highspeed digital systems. In 1987, Dr. Rutenbar received a Presidential Young Investigator Award from the National Science Foundation. He was General Chair of the 1996 International Conference on CAD. From 1992 through 1996, he chaired the Analog Technical Advisory Board for Cadence Design Systems. In 2001 he was cowinner of the Semiconductor Research Corporation's Aristotle Award for contributions to graduate education. He is a Fellow of the IEEE and a member of the ACM and Eta Kappa Nu. 